\title{
Less Is More. Cation Exchange and the Chemistry of the Nanocrystal Surface
}

\author{
Yolanda Justo, ${ }^{t, \neq, \S, *}$ Laxmi Kishore Sagar, ${ }^{\dagger, \S}$ Stijn Flamee, ${ }^{t, \S}$ Qiang Zhao, ${ }^{\perp}$ André Vantomme, ${ }^{\perp}$ and \\ Zeger Hens ${ }^{\dagger, \S, *}$
}

${ }^{\dagger}$ Physics and Chemistry of Nanostructures, Ghent University, Ghent, Belgium, ${ }^{\ddagger}$ SIM SoPPoM Project, and ${ }^{\perp}$ Instituut voor Kern en Stralingsfysica, University of Leuven, Leuven, Belgium, and ${ }^{\S}$ Center for Nano and Biophotonics, Ghent University, Ghent, Belgium

\begin{abstract}
We link the extent of $\mathrm{Pb}$ for $\mathrm{Cd}$ cation exchange reactions in $\mathrm{PbS}$ colloidal quantum dots (QDs) to their surface chemistry. Using PbS QDs with either a full or a partial surface coverage by excess $\mathrm{Pb}$, we demonstrate the central role played by vacant cation sites on the QD surface. They facilitate the adsorption of cations from solution, and they act as a source of vacancies needed for the transport of cations through the crystal lattice. This model explains our finding that the cation exchange reaction runs to completion when using a low $\mathrm{Cd}$ excess in the exchange bath, while it is impeded by a high Cd excess. Whereas in the latter case, the QD surface is poisoned by surface $C d$,

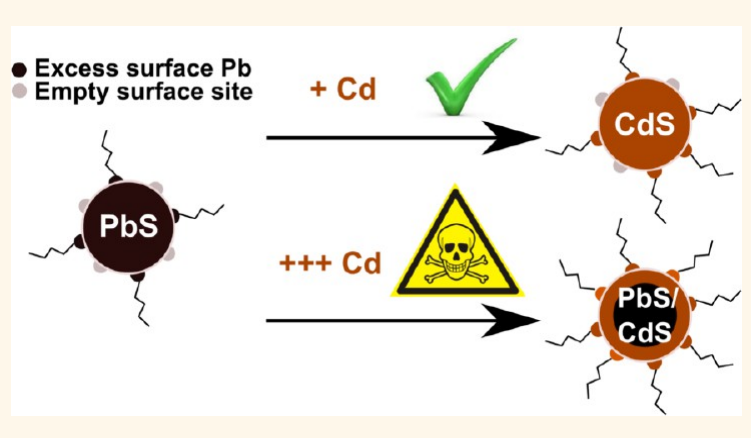
the former conditions provide the mixture of surface $\mathrm{Cd}$ and vacant surface sites the exchange reaction needs to proceed. This understanding provides a missing link needed to build a unifying mechanistic picture of cation exchange reactions at nanocrystals.
\end{abstract}

KEYWORDS: quantum dots $\cdot$ lead chalcogenides $\cdot$ cadmium chalcogenides $\cdot$ core/shell $\cdot$ surface reactions

C ation exchange has become a widely used postsynthetic method to transform colloidal nanocrystals into complex heteronanostructures, often inaccessible by other synthetic approaches. ${ }^{1,2}$ The process involves the replacement of the cations in compound nanocrystals such as metal selenides or sulfides by other cations dispersed in solution. Long known as a feasible yet slow process for bulk materials, cation exchange becomes especially attractive with nanocrystals since it gains speed, ${ }^{3}$ offers the possibility to tune the direction of the exchange by changing the coordination of cations in solution, ${ }^{4}$ and tends to preserve the anion sublattice. ${ }^{5-7}$

The complete exchange of the original by newly supplied cations has been used for the formation of homogeneous nanocrystals that proved difficult to synthesized otherwise-including III-V quantum dots, ${ }^{8}$ lanthanide fluoride nanocrystals, ${ }^{9}$ and various quantum rods ${ }^{4,6,10}$ _and heterostructures based on a heterogeneous anion sublattice, where CdSe/CdS dot-in-rod nanostructures proved a convenient starting point. ${ }^{5,11}$ The partial exchange of the original cations on the other hand leads to heteronanostructures with a heterogeneous cation sublattice. The most straightforward example involves core/shell dot-in-dot structures such as $\mathrm{PbX} / \mathrm{CdX}(\mathrm{X}=\mathrm{S}$, Se, or $\mathrm{Te}){ }^{12,13} \mathrm{CulnS}_{2} / \mathrm{ZnS}^{14}$ and InP/GaP/ZnS, ${ }^{15}$ where shell growth is typically used to enhance photoluminescence quantum yields. More intriguing is the formation of rod-like structures featuring multiple, approximately equally spaced dots such as $\mathrm{Ag}_{2} \mathrm{~S}-\mathrm{CdS}^{16}$ and PbS-CdS. ${ }^{17}$ Alternatively, when the two phases formed during the exchange are miscible, partial cation exchange may result in alloyed nanocrystals such as $(\mathrm{Cd}, \mathrm{Zn}) \mathrm{Se}^{18}$ and $(\mathrm{Hg}, \mathrm{Cd}) \mathrm{Te}^{19,20}$ although the distinction between a graded core/shell and a random alloy is often difficult. Further reducing the amount of cations exchanged makes possible the doping of nanocrystals with one or a few impurities, with a well-controlled doping density. ${ }^{21,22}$ In a somewhat different context, cation exchange has also been used during synthesis, where initially formed SnSe nuclei mediated the highly efficient formation of PbSe nanocrystals ${ }^{23}$ and $\mathrm{Cd}$ chalcogenide octapods were synthesized by means of an in situ formed $\mathrm{Cu}_{2-x}$ Se nanocrystal. ${ }^{24}$

Despite the increasing interest in cation exchange in the field of colloidal nanocrystals, little is known about the overall

\section{* Address correspondence to Yolanda.Justo@UGent.be;} Zeger.Hens@UGent.be.

Received for review April 4, 2014 and accepted August 4, 2014.

Published online August 04, 2014 $10.1021 /$ nn5037812

(c) 2014 American Chemical Society 
exchange mechanism and its different constituent steps. Thermodynamic considerations are often used to analyze the feasibility of an exchange reaction, ${ }^{1,2}$ yet only in the case of rapidly exchanging systems such as $\mathrm{Ag}_{2} \mathrm{~S}-\mathrm{CdS}$ and $\mathrm{Cu}_{2} \mathrm{~S}-\mathrm{CdS}$ could the morphology of the heteronanostructures formed be deduced from free energy arguments, including interfacial and strain contribution. ${ }^{16}$ For slower reactions such as $\mathrm{PbX} / \mathrm{CdX}$, a quasi-continuum model based on solid-state diffusion of cations has been proposed, ${ }^{25}$ while the strongly temperature-dependent kinetics and the anisotropy of the exchange has been interpreted in terms of vacancy-assisted diffusion. ${ }^{7,26,27}$ These studies, however, only address the possible mechanism of a single subreaction, while the overall kinetics of a cation exchange reaction depends on the rate-and thus the mechanism-of all subreactions proceeding in a concerted manner. ${ }^{2}$ In general terms, the subreactions involve the exchange of cations between the solution and the nanocrystal surface, possibly the formation of a new phase at the nanocrystal surface, and the transport of cations through the nanocrystal to and from the reaction front. In this picture, especially the effect of the crystal surface and the passivating surface ligands on the exchange rate has remained uninvestigated, whereas this is seen as central to establishing a unifying mechanistic picture of cation exchange reactions in nanocrystals. ${ }^{1,2}$

Here, we investigate the interplay between the surface chemistry and the cation exchange reaction by means of the well-known cadmium for lead cation exchange in quasi-spherical PbS nanocrystals. Our starting point is two different $\mathrm{PbS}$ syntheses that both yield nanocrystals with the same oleate ligands, yet with a different surface excess of $\mathrm{Pb}^{28}$ The first type has $\mathrm{a} \mathrm{Pb}$ excess that corresponds to full surface coverage by excess cations, similar to what was found with $\mathrm{PbSe}$ nanocrystals, ${ }^{29}$ whereas the second type has only a partial surface coverage by excess $\mathrm{Pb}$. For both nanocrystals, we monitor the thickness of the CdS shell formed after a fixed exchange time as a function of temperature and $\mathrm{Cd}$ excess. Two observations stand out. While no exchange is observed for $\mathrm{PbS}$ quantum dots (QDs) with full excess $\mathrm{Pb}$ coverage at room temperature, the PbS QDs with partial surface coverage exhibit a self-limiting adsorption of $\mathrm{Cd}$, thereby replacing surface-adsorbed $\mathrm{Pb}$. At more elevated temperature, the exchange reaction leads to a similar limiting shell thickness for both QDs when using a high $\mathrm{Cd}$ excess, yet the exchange runs to completion when the $N_{\mathrm{Cd}}: N_{\mathrm{Pb}}$ ratio in the exchange bath is strongly reduced. These observations give evidence of the key role played by vacant cation adsorption sites at the nanocrystal surface in cation exchange reactions. They facilitate the adsorption of cations from solution and they act as a source of vacancies needed for the transport of cations through the crystal lattice. In this respect, a high $\mathrm{Cd}$ excess in the exchange bath poisons the QD surface by fully covering it by excess cations and thus impeding further exchange of cations. To the best of our knowledge, this is the first demonstration that the surface reaction can indeed limit the rate of a cation exchange reaction in colloidal nanocrystals and that a fine-tuning of the nanocrystal surface composition can significantly enhance cation exchange rates.

\section{RESULTS}

The Starting Point: PbS Quantum Dots and PbS/CdS Formation. PbS QDs were synthesized by means of two different hot injection procedures. The first has been described by Cademartiri et al. ${ }^{30}$ and Moreels et al. ${ }^{28}$ It makes use of $\mathrm{PbCl}_{2}$ and elemental sulfur, dissolved in oleylamine as precursors, and results in Pb-rich particles with a surface terminated by chloride and oleate. ${ }^{28}$ The second is based on the procedure proposed by Abel et al., ${ }^{31}$ where lead(II) oleate and bis(trimethylsilyl) sulfide $\left((\mathrm{TMS})_{2} \mathrm{~S}\right)$ are used as the precursors, resulting in PbS QDs capped with oleate ligands as well (see Methods). The PbS QDs obtained with either method will be denoted as $\mathrm{PbS}_{\mathrm{Cl}}$ and $\mathrm{PbS}_{\mathrm{TMS}}$, respectively. From the transmission electron microscopy (TEM) images shown in Figure $1 \mathrm{a}, \mathrm{b}$, it follows that both procedures result in quasi-spherical nanocrystals with a narrow size dispersion. A far more extensive study of the shape of the nanocrystals obtained by both synthesis methods is reported in the Supporting Information (section S1). As this study shows, there are no significant differences in morphology, a fact that rules out the influence that different faceting may have on the cationic exchange process. On the other hand, both types of PbS QDs exhibit markedly different $\mathrm{Pb}: \mathrm{S}$ ratios, which were determined using Rutherford backscattering spectrometry. As shown in Figure $1 \mathrm{c}$, the $\mathrm{PbS}_{\mathrm{TMS}}$ have a $\mathrm{Pb}: \mathrm{S}$ ratio in the range 1.55-1.40 for $\mathrm{QD}$ diameters ranging from 4.0 to $6.5 \mathrm{~nm}$, whereas the $\mathrm{PbS}_{\mathrm{Cl}}$ QDs feature a considerably lower $\mathrm{Pb}$ excess of 1.25-1.30 for similar QD diameters. The black dashed line in Figure 1c represents the nonstoichiometry corresponding to a full coverage of the QD surface by $\mathrm{Pb}$ (see Supporting Information section S2). Importantly, one sees that the $\mathrm{Pb}: \mathrm{S}$ ratio of the $\mathrm{PbS}_{\mathrm{Cl}} \mathrm{QDs}$ is well below that expected for full surface coverage, while that of the $\mathrm{PbS}_{\text {TMS }} \mathrm{QDs}$ agrees with the values expected for a surface fully covered by excess $\mathrm{Pb}$. In what follows, we will make use of this significant difference in surface coverage by excess cations to study the interplay between surface chemistry and the rate of cation exchange reactions.

$\mathrm{PbS} / \mathrm{CdS}$ core/shell QDs were synthesized starting from PbS core QDs by means of a cation exchange reaction as first proposed by Pietryga et al. ${ }^{12}$ and described in more detail in ref 32 . Briefly, cadmium oleate is added to a heated suspension of PbS QDs in toluene in an amount calculated relative to the number of $\mathrm{Pb}$ atoms initially present in the sample. After the 
a

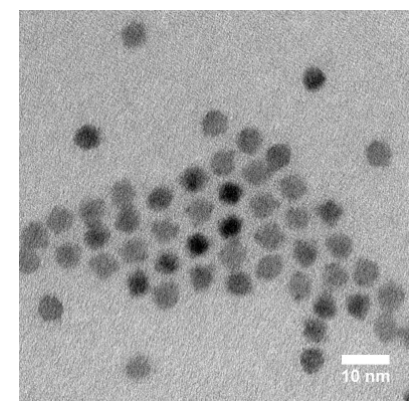

b

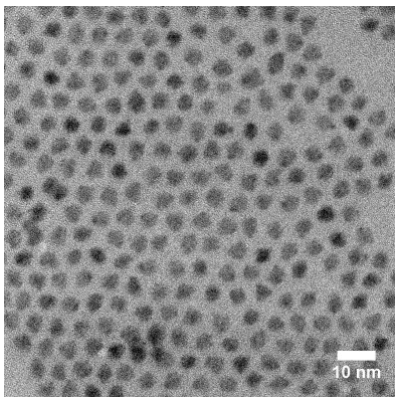

C

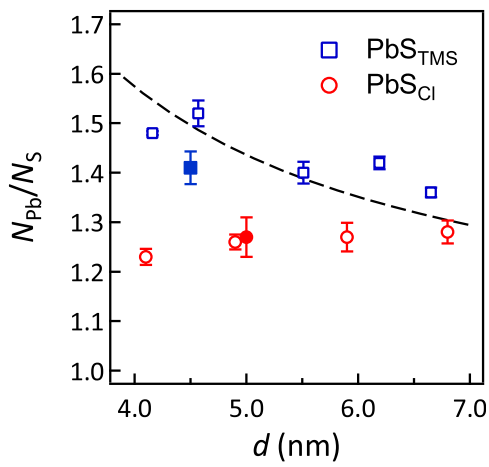

Figure 1. (a, b) TEM micrographs of the $\mathrm{PbS}_{\mathrm{Cl}}$ and $\mathrm{PbS}_{\mathrm{TMS}}$ quantum dots used in this study. (c) $\mathrm{Pb}: \mathrm{S}$ ratio for (blue squares) $\mathrm{PbS}_{\mathrm{TMS}}$ and (red circles) $\mathrm{PbS}_{\mathrm{Cl}} \mathrm{QDs}$ as a function of the diameter of the QDs. The dashed line depicts the theoretical nonstoichiometry that corresponds to a full surface coverage by excess $\mathrm{Pb}$. The filled markers indicate the $\mathrm{Pb}: \mathrm{S}$ ratio measured on the PbS QD samples used here for studying cationic exchange.
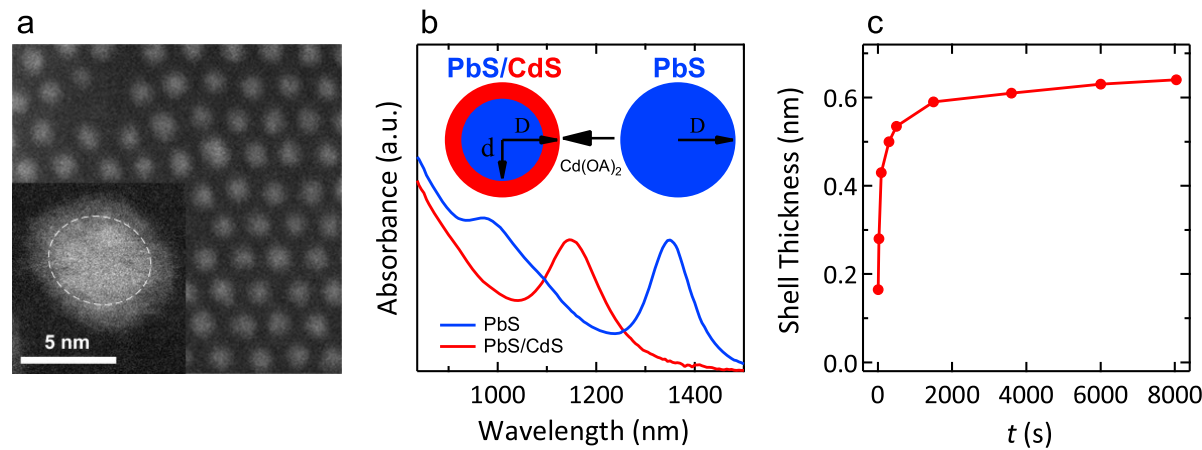

Figure 2. (a) Scanning TEM micrographs of PbS/CdS core/shell QDs recorded by high-angle annular dark field (HAADF) detection. Especially in the micrograph shown in the inset, the brighter region inside the QDs encircled by the dashed line points toward a core/shell structure with heavier elements in the core (i.e., Pb) and lighter elements in the shell (i.e., $\mathrm{Cd}$ ). Exchange reaction run at $130^{\circ} \mathrm{C}$ for $1 \mathrm{~h}$ and a $N_{\mathrm{Cd}}: N_{\mathrm{Pb}}$ ratio of 20:1 starting from $5.0 \mathrm{~nm}$ PbS QDs. (b) Example of absorption spectra of (blue) PbS core QDs and (red) PbS/CdS core/shell QDs formed out of it. The inset shows the concentric core/shell model used to determine an effective core diameter from the peak wavelength of the first exciton transition. (c) Evolution of the shell thickness as a function of time for $5 \mathrm{~nm} \mathrm{PbS} / \mathrm{Cl} / \mathrm{CdS} \mathrm{QDs}$. Exchange reaction run at $100{ }^{\circ} \mathrm{C}$ and a $N_{\mathrm{Cd}}: N_{\mathrm{Pb}}$ ratio of $20: 1$.

desired reaction time, the reaction is quenched and the obtained PbS/CdS QDs are purified twice using methanol as the nonsolvent and stored in toluene. As demonstrated by various authors, exposure of PbS QDs to cadmium oleate initiates the replacement of the outermost $\mathrm{Pb}$ atoms in the QDs by $\mathrm{Cd}$, which results in the formation of a $\mathrm{CdS}$ shell around a remaining $\mathrm{PbS}$ core of reduced size as confirmed by the bright/dark contrast in the scanning TEM image shown in Figure $2 a$. Importantly the cation exchange reaction does not alter the overall morphology of the original PbS QDs under the conditions used in this study (see Supporting Information section S1). Under this condition, the exchange reaction can be monitored by means of absorption spectroscopy, where the shrinking of the core and the concomitant increase of quantum confinement lead to a blue shift of the first absorption peak related to the first exciton or band gap transition.

As shown previously, the diameter of the PbS core in $\mathrm{PbS} / \mathrm{CdS}$ core/shell QDs can be determined from the peak wavelength of the first exciton transition using the sizing curve established for PbS QDs, ${ }^{32}$ unlike other core/shell systems such as PbSe/PbS QDs, where the wave function of the charge carriers are delocalized over the entire core/shell structure. ${ }^{33,34}$ As the total particle size coincides with that of the parent PbS QDs, this means that also an effective or net shell thickness can be readily calculated when assuming a spherical and concentric core/shell QD (Figure 2b). Figure 2c depicts the evolution of the shell thickness thus determined as a function of reaction time for $5 \mathrm{~nm} \mathrm{PbS}$ QDs exposed to a cadmium oleate solution at $100{ }^{\circ} \mathrm{C}$ and a $N_{\mathrm{Cd}}: N_{\mathrm{Pb}}$ ratio of 20:1 (Cd added versus Pb initially present). The graph makes clear that the shell thickness increases rapidly at the beginning of the reaction and levels off after $\sim 2000 \mathrm{~s}$. In this work, we therefore studied the effect of different reaction parameters (type of $\mathrm{QD}$, temperature, $N_{\mathrm{Cd}}: N_{\mathrm{Pb}}$ ratio) by stopping the cation exchange after $1 \mathrm{~h}$ of reaction time and analyzing the thickness of the resulting $\mathrm{CdS}$ shell using absorption spectroscopy.

Cation Addition versus Cation Exchange. Figure 3a shows the effective shell thickness obtained from the analysis of the absorption spectrum for exchange reactions carried out at room temperature using both $\mathrm{PbS}_{\mathrm{Cl}}$ and $\mathrm{PbS}_{\text {TMS }} \mathrm{QDs}$. In the case of $\mathrm{PbS}_{\text {TMS }}$ QDs, no noticeable 

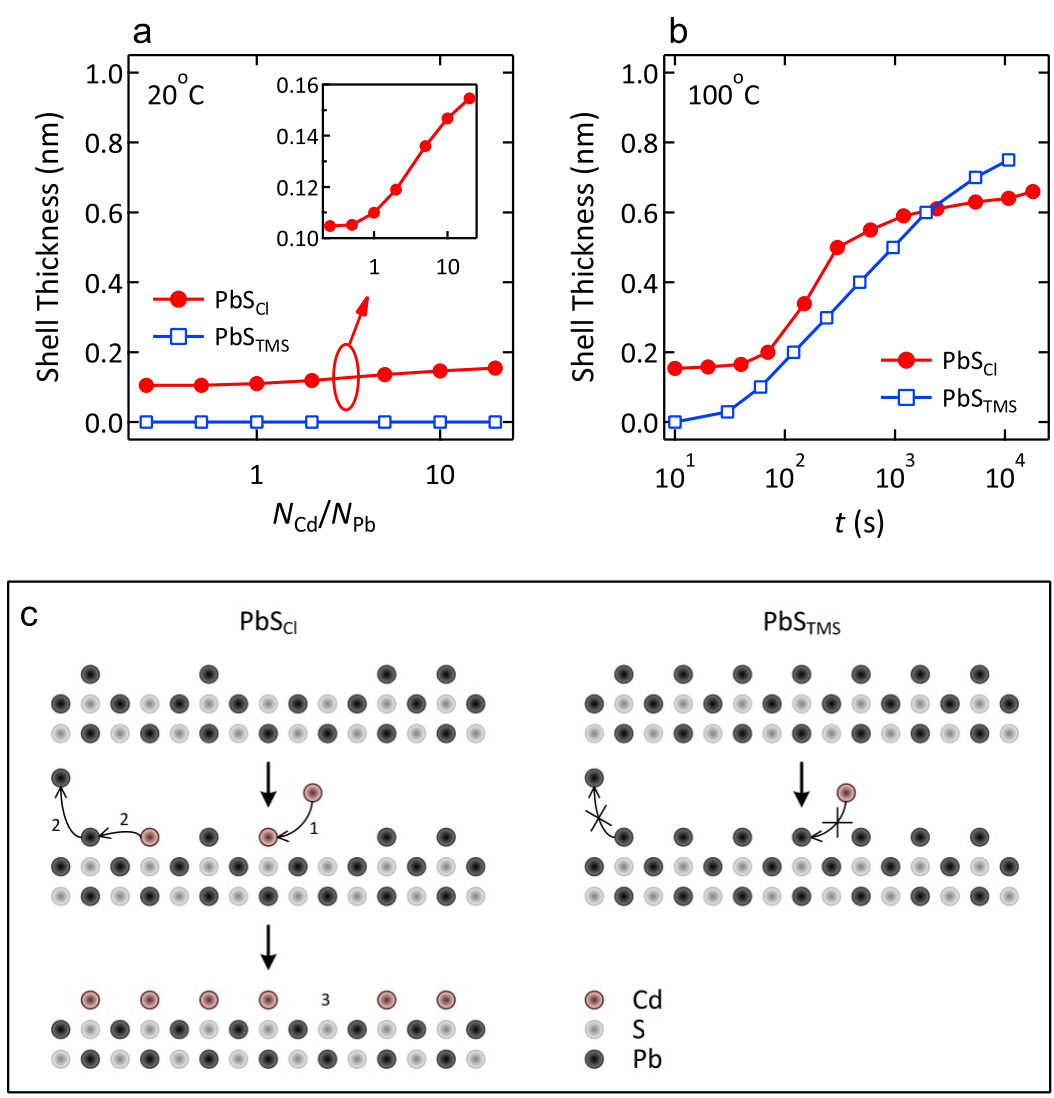

Figure 3. (a) Shell thickness obtained with (open blue squares) $\mathrm{PbS}_{\mathrm{TMS}}$ and (filled red circles) $\mathrm{PbS}_{\mathrm{Cl}}$ core QDs kept for $1 \mathrm{~h}$ in exchange baths with different $N_{\mathrm{Cd}}: N_{\mathrm{Pb}}$ ratios at $20^{\circ} \mathrm{C}$. The inset shows more clearly the variation in shell thickness for the $\mathrm{PbS}_{\mathrm{Cl}}$ QDs. (b) Time evolution of the shell thickness for (open blue squares) $\mathrm{PbS}_{\mathrm{TMS}}$ and (filled red circles) $\mathrm{PbS}_{\mathrm{Cl}}$ core QDs during an exchange reaction at $100^{\circ} \mathrm{C}$ with $N_{\mathrm{Cd}}: N_{\mathrm{Pb}}=20: 1$. (c) Cartoon representation of possible steps in the exchange of $\mathrm{Pb}$ for $\mathrm{Cd}$ on (left) $\mathrm{PbS}_{\mathrm{Cl}}$ and (right) $\mathrm{PbS}_{\mathrm{TMS}}$ surfaces, featuring (1) the adsorption of cadmium species on empty surface sites, (2) the removal of surface $\mathrm{Pb}$ by surface $\mathrm{Cd}$, and ( 3 ) a remaining empty surface site in the initially formed $\mathrm{CdS}$ shell.

CdS shell is formed, even if the excess of cadmium oleate is raised to a 20:1 ratio relative to the amount of lead present. On the other hand, a slight shift in absorbance is seen in the case of $\mathrm{PbS}_{\mathrm{Cl}} \mathrm{QDs}$, which can be translated into a CdS shell with a thickness increasing from 0.1 to almost $0.16 \mathrm{~nm}$ when the $N_{\mathrm{Cd}}$ : $N_{\mathrm{Pb}}$ ratio increases from $0.25: 1$ to $20: 1$. To investigate this difference further, we raised the temperature of the exchange reaction using an $N_{\mathrm{Cd}}: N_{\mathrm{Pb}}$ ratio of 20:1 and monitored the shell thickness as a function of time. As shown in Figure 3b, a difference in shell thickness for both types of QDs similar to what was observed at room temperature now appears in the early stages of the exchange process. Whereas for $\mathrm{PbS}_{\text {TMS }} \mathrm{QDs}$, hardly any CdS shell is formed after $10 \mathrm{~s}, \mathrm{PbS}_{\mathrm{Cl}}$ QDs already have a $0.16 \mathrm{~nm}$ thick CdS shell. For the latter QDs, this thickness is maintained during the first $60 \mathrm{~s}$ of the reaction, after which it grows at a rate similar to that of the $\mathrm{PbS}_{\mathrm{TMS}} \mathrm{QDs}$. In both cases, the shell growth slows down with increasing reaction time to reach a thickness of 0.65 to $0.75 \mathrm{~nm}$ after $1 \mathrm{~h}$ of reaction.

Given the difference in excess $\mathrm{Pb}$ on the surface of both types of QDs-with full coverage in the case of $\mathrm{PbS}_{\text {TMS }} \mathrm{QDs}$ and partial coverage with $\mathrm{PbS}_{\mathrm{Cl}} \mathrm{QDs}-\mathrm{a}$ first interpretation of the fast initial shell formation observed with $\mathrm{PbS}_{\mathrm{Cl}}$ is that of a rapid adsorption of cadmium oleate on surface sites not occupied by excess $\mathrm{Pb}$, as represented by step 1 in Figure 3c. This could then facilitate the exchange of $\mathrm{Pb}$ for $\mathrm{Cd}$ on the QD surface (step 2 in Figure 3c), resulting in the formation of an initial $\mathrm{CdS}$ shell where-given the PbS lattice parameter of $0.59 \mathrm{~nm}$ - the thickness of $\sim 0.16 \mathrm{~nm}$ indeed corresponds to the replacement of a submonolayer of $\mathrm{Pb}$ by $\mathrm{Cd}$. In the case of $\mathrm{PbS}_{\mathrm{TMS}}$, the lack of these empty surface sites prevents this adsorption of $\mathrm{Cd}$ species and thus the rapid formation of an initial CdS shell.

A quantitative argument that supports this interpretation comes from a comparison of the shell thickness of $\mathrm{PbS} / \mathrm{CdS}$ QDs, calculated either from the $\mathrm{Pb}: \mathrm{Cd}$ ratio as determined using elemental analysis by inductively coupled plasma optical emission spectrometry (ICP-OES) or from the shift of the first exciton transition in the absorption spectrum. Since the PbS core in $\mathrm{PbS} / \mathrm{CdS}$ QDs has the same sizing curve as PbS coreonly QDs, the shift of the first exciton peak in the absorption spectrum is determined by the amount of $\mathrm{Cd}$ cations that effectively replace $\mathrm{Pb}$ cations, and not by $\mathrm{Cd}$ cations occupying previously empty surface 
sites. On the other hand, elemental analysis by ICP-OES measures all the $\mathrm{Cd}$ present in the system. Figure 4 compares the shell thickness determined by both methods for $\mathrm{PbS}_{\text {TMS }}$ (Figure $4 \mathrm{a}$ ) and $\mathrm{PbS}_{\mathrm{Cl}}$ (Figure $4 \mathrm{~b}$ ) exposed at $100^{\circ} \mathrm{C}$ to cadmium oleate with a $0.5: 1$ and a 20:1 $N_{C d}: N_{\mathrm{Pb}}$ excess. The respective exchange times of 2 and $1 \mathrm{~min}$ were chosen to obtain similar shell thicknesses for either of the two resulting $\mathrm{PbS} / \mathrm{CdS}$ QDs. It follows that in the case of $\mathrm{PbS}_{\mathrm{TMS}} \mathrm{QDs}$ both methods yield the same shell thickness, meaning that each $\mathrm{Cd}$ atom in the system has effectively replaced a $\mathrm{Pb}$ atom from the parent $\mathrm{PbS} \mathrm{QDs}$. On the other hand, ICP-OES systematically leads to a larger shell thickness than the analysis of the absorption spectrum in the case of $\mathrm{PbS}_{\mathrm{Cl}} \mathrm{QDs}$. Hence, these systems also contain $\mathrm{Cd}$ atoms that were added to the parent PbS QDs, without replacing $\mathrm{Pb}$ atoms originally present. Moreover, one sees that the difference between both methods is larger for the larger excess, a result in line with the idea of a more complete occupation of the empty surface sites by cadmium species when the cadmium oleate concentration is higher. We thus conclude that vacant surface sites play a key role in the initial steps of a cation exchange reaction. They make
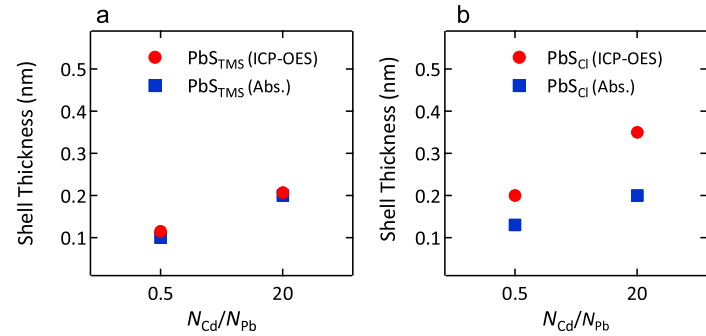

Figure 4. Comparison between the shell thickness obtained by ICP-OES analysis (filled red circles) and that obtained using the PbS QD sizing curve (filled blue squares) for (a) $\mathrm{PbS}_{\mathrm{TMS}} \mathrm{QDs}$ after $2 \mathrm{~min}$ reaction and (b) $\mathrm{PbS}_{\mathrm{Cl}}$ QDs after $1 \mathrm{~min}$ reaction. Exchange carried out at $100{ }^{\circ} \mathrm{C}$.

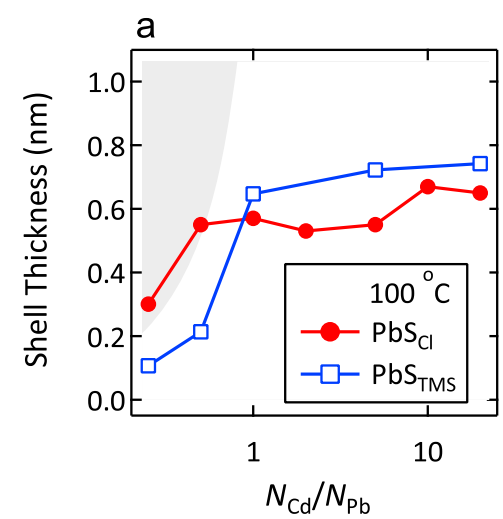

possible the adsorption of cadmium oleate on the QD surface and thus facilitate the replacement of surface $\mathrm{Pb}$ cations by $\mathrm{Cd}$ cations. Moreover, the occupation of the vacant surface sites by cadmium species depends on the concentration of cadmium oleate in the exchange bath, where higher concentrations lead to a more complete surface coverage. This conclusion is in line with the findings reported by Anderson et al., ${ }^{35}$ who show that the cadmium carboxylate complexes that make up the cation surface excess in the case of CdSe QDs should be seen as removable Z-type ligands that can be involved in an adsorption/desorption equilibrium.

Full Yield Exchange of Cations. As already shown in Figures 2 and $3 \mathrm{~b}$, after reaction times of $1 \mathrm{~h}$ or more, the shell thickness levels off to an approximately steady value. In Figure 5a, we plot the shell thickness obtained after $1 \mathrm{~h}$ of reaction time at $100{ }^{\circ} \mathrm{C}$ as a function of the $N_{\mathrm{Cd}}: N_{\mathrm{Pb}}$ ratio for both types of QDs. One sees that once the $N_{\mathrm{Cd}}: N_{\mathrm{Pb}}$ ratio exceeds 1 , an almost constant thickness in the range $0.6-0.8 \mathrm{~nm}$ is obtained in both cases. The slight difference between both types of PbS QDs could be explained by the difference of $0.5 \mathrm{~nm}$ in initial diameter. From experience, we know that larger QDs get thinner shells under similar exchange conditions. For an $N_{\mathrm{Cd}}: N_{\mathrm{Pb}}$ ratio smaller than 1 on the other hand, the thickness of the CdS shell obtained with $\mathrm{PbS}_{\mathrm{Cl}}$ QDs systematically exceeds that of the $\mathrm{PbS}_{\text {TMS }} \mathrm{QDs}$. For comparison, we added to Figure $5 \mathrm{a}$ a filled gray region that indicates the shell thicknesses out of reach for $\mathrm{PbS}_{\mathrm{Cl}} / \mathrm{CdS} \mathrm{QDs}$, given the $N_{\text {Cd }}: N_{\mathrm{Pb}}$ ratio used. The lower boundary of this region corresponds to the maximum shell thickness $\delta_{\max }$ that is attained when the exchange is full yield either in cadmium $\left(N_{\mathrm{Cd}}: N_{\mathrm{Pb}}<1\right)$ or in lead $\left(N_{\mathrm{Cd}}: N_{\mathrm{Pb}}>1\right)$ (see Supporting Information section S3). In the former case, this results in $\mathrm{PbS} / \mathrm{CdS}$ core/shell QDs where the average ratio of the shell volume to the total QD

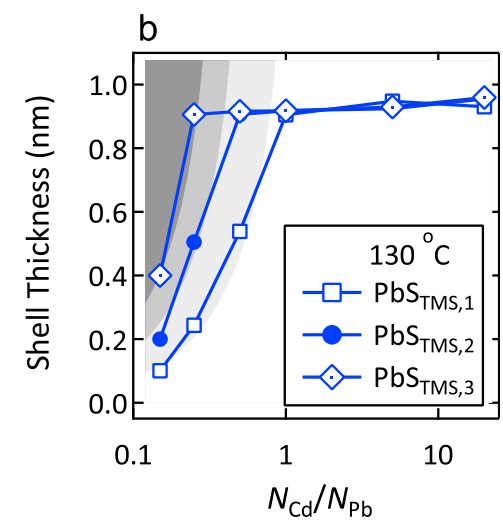

Figure 5. (a) Shell thickness obtained after $1 \mathrm{~h}$ of exchange at $100^{\circ} \mathrm{C}$ as a function of the $N_{\mathrm{Cd}}: N_{\mathrm{Pb}}$ ratio for (red circles) $\mathrm{PbS}$ Cl and (open blue squares) $\mathrm{PbS}_{\mathrm{TMS}} \mathrm{QDs}$. The gray region indicates the shell thickness out of reach for the $\mathrm{PbS}_{\mathrm{Cl}} \mathrm{QDs}$. The comparable region for the $\mathrm{PbS}_{\text {TMS }} \mathrm{QDs}$ is nearly identical and therefore not indicated. (b) Shell thickness obtained after (open blue squares) single, (filled blue circles) double, and (dotted blue diamonds) triple exchange for $1 \mathrm{~h}$ at $130{ }^{\circ} \mathrm{C}$ as a function of the $N_{\mathrm{Cd}}: N_{\mathrm{Pb}}$ ratio for $\mathrm{PbS}_{\text {TMS }} \mathrm{QDs}$. The gray regions indicate the shell thickness out of reach for (dark gray) triple exchange, (dark gray + gray) double exchange, and (dark gray + gray + light gray) single exchange. 

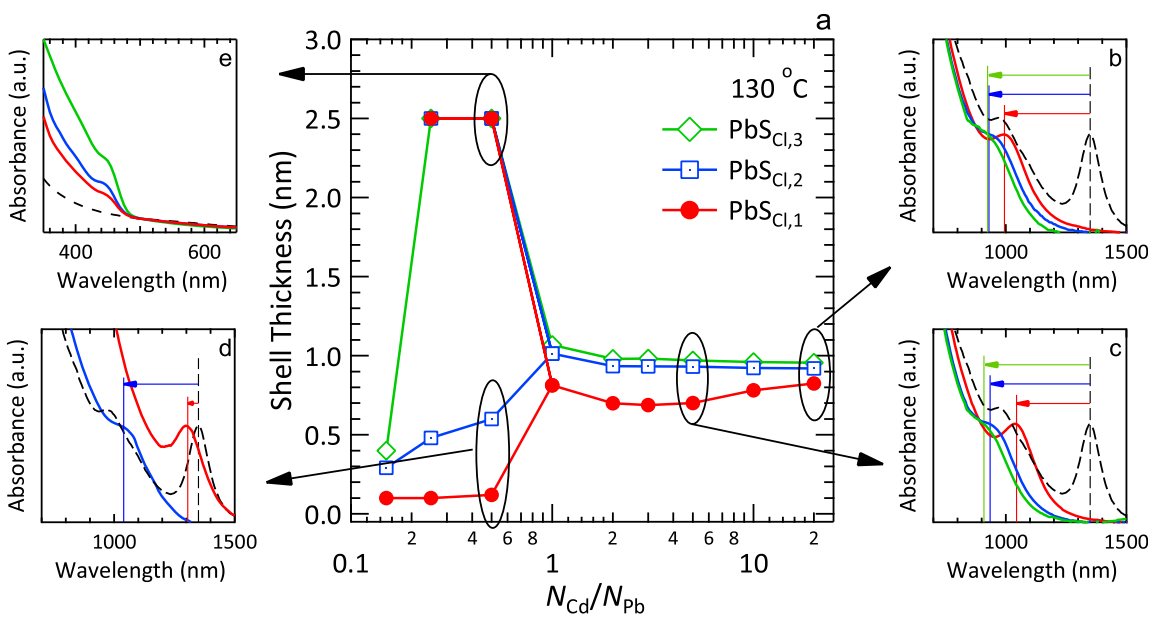

Figure 6. (a) Evolution of the shell thickness for three successive cation exchange reactions carried out at $130^{\circ} \mathrm{C}$, as a function of $N_{\mathrm{Cd}}: N_{\mathrm{Pb}}$ ratio for $\mathrm{PbS}_{\mathrm{Cl}} \mathrm{QDs}$. (b) Absorption spectra of (dashed black line) the original $\mathrm{PbS} \mathrm{Cl}_{\mathrm{Cl}} \mathrm{QDs}$ and the resulting $\mathrm{PbS} / \mathrm{CdS}$ QDs after (red) one, (blue) two, and (green) three exchange cycles for an $N_{\mathrm{Cd}}: N_{\mathrm{Pb}}$ ratio of 20 . The lines indicate the shift of the main absorption feature. (c) The same for an $N_{\mathrm{Cd}}: N_{\mathrm{Pb}}$ ratio of 5. (d) The same for an $N_{\mathrm{Cd}}: N_{\mathrm{Pb}}$ ratio of 0.5 . (e) Visible part of the absorption spectrum after the various exchange steps, normalized at $\lambda=500 \mathrm{~nm}$.

volume equals the $N_{\mathrm{Cd}}: N_{\mathrm{Pb}}$ ratio used in the synthesis - a situation we will refer to as Cd-limited full yield exchange-whereas the latter case corresponds to the complete transformation of each $\mathrm{PbS}$ $\mathrm{QD}$ into a CdS QD, i.e., Pb-limited full yield exchange. The corresponding region for $\mathrm{PbS}_{\mathrm{TMS}} / \mathrm{CdS} \mathrm{QDs}$ is almost identical and, therefore, not shown. The figure indicates that for $\mathrm{PbS}_{\text {TMS }} \mathrm{QDs}$ the exchange is far from complete for all $N_{\mathrm{Cd}}: N_{\mathrm{Pb}}$ ratios considered since in both the $\mathrm{Cd}$ - and the $\mathrm{Pb}$-limited regime the shell thickness attained remains well below $\delta_{\text {max }}$. In the case of $\mathrm{PbS}_{\mathrm{Cl}}$ QDs on the other hand, the obtained shell thickness is lower than $\delta_{\text {max }}$ at high $N_{\mathrm{Cd}}: N_{\mathrm{Pb}}$ ratios, yet it coincides with $\delta_{\text {max }}$ for the two lowest $N_{\mathrm{Cd}}: N_{\mathrm{Pb}}$ ratios of 0.5 and 0.25 . In other words, the number of $\mathrm{Pb}$ cations exchanged remains well below what is possible when using a high $N_{\mathrm{Cd}}: N_{\mathrm{Pb}}$ ratio, while it amounts to its maximum possible value at low $N_{\mathrm{Cd}}: N_{\mathrm{Pb}}$ ratios.

To investigate this apparently contradictory outcome in more detail, we further raised the exchange temperature to $130^{\circ} \mathrm{C}$, analyzing once more the shell thickness obtained after $1 \mathrm{~h}$ of reaction time using absorption spectroscopy. Moreover, after stopping the exchange reaction after $1 \mathrm{~h}$ and measuring the absorption spectrum of the resulting $\mathrm{PbS} / \mathrm{CdS}$ QDs, we restarted the exchange process a second time using a new solution of cadmium oleate with the same initial $N_{\mathrm{Cd}}: N_{\mathrm{Pb}}$ ratio and, after analysis of the core/shell QDs, a third time to monitor the further development of the shell thickness. In the case of $\mathrm{PbS}_{\text {TMS }} \mathrm{QDs}$, we again find that for $N_{\mathrm{Cd}}: N_{\mathrm{Pb}}$ ratios larger than 1 the shell thickness reaches a steady value of $0.9-1.0 \mathrm{~nm}$, i.e., slightly larger than when using $100{ }^{\circ} \mathrm{C}$ (see Figure 5b). Reexposing the $\mathrm{PbS} / \mathrm{CdS}$ QDs to a fresh exchange bath for these $N_{\text {Cd }}: N_{\mathrm{Pb}}$ ratios does not increase the shell thickness any further. For $N_{\mathrm{Cd}}: N_{\mathrm{Pb}}<1$ however, we find also in this case that the shell thickness corresponds to $\delta_{\max }$ after a first exchange step; that is, a situation of Cd-limited full yield exchange is now also reached for $\mathrm{PbS}_{\mathrm{TMS}}$ QDs. Moreover, for the two smallest $N_{\mathrm{Cd}}: N_{\mathrm{Pb}}$ ratios used ( 0.15 and 0.25$)$, the exchange reaction reaches $C d$-limited full yield also in the second and third exchange step.

The difference between low and high $N_{\mathrm{Cd}}: N_{\mathrm{Pb}}$ ratios on the cation exchange becomes even more pronounced when looking at the shell thickness of $\mathrm{PbS}_{\mathrm{Cl}} / \mathrm{CdS} \mathrm{QDs}$ during the same sequence of exchange reactions. Figure 6 summarizes the evolution of the shell thickness for the different exchange steps as a function of the $N_{\mathrm{Cd}}: N_{\mathrm{Pb}}$ ratio and illustrates these with a number of representative absorption spectra. As shown in Figure $6 b, c$, the absorption spectra of $\mathrm{PbS}_{\mathrm{Cl}}$ QDs after $1 \mathrm{~h}$ of cation exchange under conditions where $N_{\mathrm{Cd}}: N_{\mathrm{Pb}}$ is equal to or larger than 1 show an approximately constant blue shift of the first exciton, corresponding to a shell thickness of $0.7-0.8 \mathrm{~nm}$. On the other hand, the use of an $N_{\mathrm{Cd}}: N_{\mathrm{Pb}}$ ratio smaller than 1 leads to some broadening of the first exciton absorption and a small spectral blue shift that corresponds to a CdS shell thickness of only $\sim 0.1 \mathrm{~nm}$ (see Figure $6 \mathrm{~d}$ ), i.e., well below $\delta_{\max }$ for $N_{\mathrm{Cd}}: N_{\mathrm{Pb}}$ ratios of 0.5 and 0.25 . However, as shown in Figure $6 e$, an additional pronounced absorption feature appears at the same time at wavelengths of around $450 \mathrm{~nm}$ for $N_{\text {Cd }}: N_{\mathrm{Pb}}$ ratios of 0.5 and 0.25 . As argued in the Supporting Information (section S4), PbS/CdS QDs with a thin CdS shell have an absorption coefficient that is nearly identical to that of PbS QDs in this wavelength range. We therefore attribute this additional feature to the formation of CdS QDs by the complete transformation of part of the original PbS QDs. We examined this premise further by means of energy dispersive X-ray spectroscopy coupled to a scanning TEM (STEM-EDX). The results, summarized in the Supporting Information (section S5), confirm that individual QDs on a single 


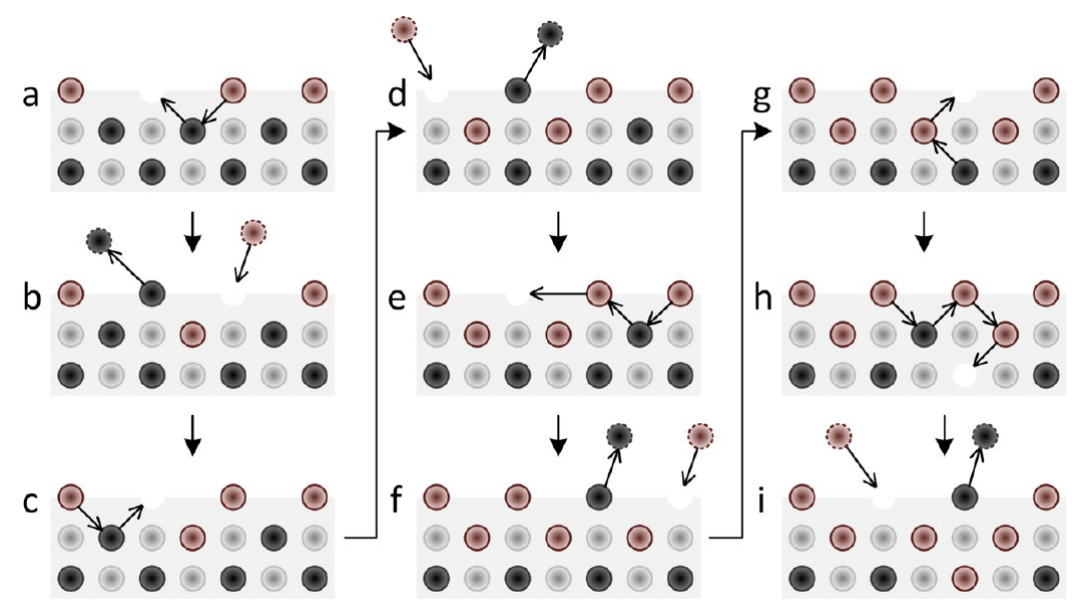

Figure 7. Cartoon representation of different exchange steps involved in the successive formation of Cd-containing lattice planes. $(\mathrm{a}, \mathrm{c})$ Transfer of a Pb cation to an empty surface site followed by hopping of a surface $\mathrm{Cd}$ cation into the thus created vacancy. (b, $d, f, i)$ Adsorption of a Cd complex from solution followed by the desorption of a surface $\mathrm{Pb}$ complex. (e) Diffusion of a surface Cd cation on the QD surface, followed by the process depicted in (a) and (c). (g) Transfer of a Cd cation to an empty surface site followed by hopping of a $\mathrm{Pb}$ cation from the underlying lattice plane into the thus created vacancy. (h) Sequential hopping steps induced by a deep vacancy, transferring $\mathrm{Cd}$ cations to the reaction front and $\mathrm{Pb}$ cations to the QD surface.

TEM grid either show mainly $\mathrm{Pb}$ and S signals (Figure S3) in line with the minor shift of the first exciton transitionor are fully converted into $\mathrm{CdS}$, whereas the presence of $\mathrm{Pb}, \mathrm{Cd}$, and $\mathrm{S}$ can be detected in an ensemble of QDs.

In Figure 6e, fully converted CdS QDs are represented by a shell thickness of $2.5 \mathrm{~nm}$, i.e., the radius of the original $\mathrm{PbS}_{\mathrm{Cl}} \mathrm{QDs}$. The presence of both $\mathrm{PbS} / \mathrm{CdS}$ QDs and CdS QDs in the same samples is expressed accordingly by indicating two shell thicknesses. Importantly, the fraction of PbS QDs completely transformed into $\mathrm{CdS}$ is not negligible. The spectra shown in Figure 6e have been normalized at $500 \mathrm{~nm}$, a wavelength where CdS QDs are still transparent. At shorter wavelengths, the contribution of the $\mathrm{PbS} / \mathrm{CdS}$ QDs to the absorbance can be estimated from the dashed line, which gives the original $\mathrm{PbS}$ absorbance normalized at $500 \mathrm{~nm}$, since the absorption coefficient of $\mathrm{PbS} / \mathrm{CdS}$ QDs is about equal to that of PbS QDs for thin shells (see Supporting Information, section S4). The difference between the absorbance of the exchanged samples and the dashed line therefore measures the contribution of the CdS QDs to the absorbance. It follows that after the first exchange step with an $N_{\mathrm{Cd}}$ : $N_{\mathrm{Pb}}$ ratio of 0.5 both fractions have an about equal contribution to the absorbance at wavelengths shorter than $400 \mathrm{~nm}$. Although an exact quantification is not possible, as the absorption coefficient of the CdS QDs thus formed is unknown, this indicates that the fractions of QDs fully transformed and hardly affected are comparable (see Supporting Information section S6) and that the complete transformation stops when the reaction reaches full yield in $\mathrm{Cd}$.

The different exchange behavior for low and high $N_{\text {Cd }}: N_{\mathrm{Pb}}$ ratios is only enlarged by re-exposing the $\mathrm{PbS} / \mathrm{CdS}$ QDs to the exchange bath. When $N_{\mathrm{Cd}}: N_{\mathrm{Pb}}>1$, a limiting shell thickness of $0.9-1.0 \mathrm{~nm}$ is obtained that hardly increases when repeating the exchange reaction. For $N_{\mathrm{Cd}}: N_{\mathrm{Pb}}$ ratios of 0.5 and 0.25 , the increasing intensity of the CdS-related absorption feature indicates that an ever larger portion of the QDs is fully transformed into CdS after the second and third exposure. Moreover, although the use of an $N_{\mathrm{Cd}}: N_{\mathrm{Pb}}$ ratio of 0.15 does not lead to full transformation, the shell thickness obtained after 3 cycles is close to the maximum value it can attain for the total amount of $\mathrm{Cd}$ used during the three successive exchange reactions. The exchange reaction thus attains $\mathrm{Cd}$-limited full yield also for this low $N_{\mathrm{Cd}}: N_{\mathrm{Pb}}$ ratio.

\section{DISCUSSION}

The observation that cation exchange is facilitated by low $N_{\mathrm{Cd}}: N_{\mathrm{Pb}}$ ratios and can even lead-in strong contrast to exchange reactions with a high Cd excessto the complete transformation of PbS into CdS can be understood based on the partial surface termination of the QDs by excess cations. It has been argued before that lattice vacancies are needed for $\mathrm{Cd}$ and $\mathrm{Pb}$ cations to diffuse through the $\mathrm{PbS}$ or $\mathrm{CdS}$ crystal lattice. Looking at Figure $3 c$, one understands that unoccupied surface sites-labeled by 3 in Figure 3c-are a source of such vacancies. In Figure 7, we take the $\mathrm{PbS}_{\mathrm{Cl}} \mathrm{QDs}$ with their initially formed Cd-terminated surface as a starting point to show that the presence of unoccupied surface sites not only enables adsorption of $\mathrm{Cd}$ species but also provides the mobility that is needed to bring $\mathrm{Pb}$ atoms from the inside to the surface of the QDs and replace them by $\mathrm{Cd}$ atoms. Figure $7 \mathrm{a}$ depicts a process where by sequential hopping a $\mathrm{Pb}$ atom moves to the $\mathrm{PbS}$ surface, whereas the vacant site it leaves behind is occupied by a $\mathrm{Cd}$ atom initially present at the surface. By a process similar to that shown in Figure $3 c$, the resulting surface $\mathrm{Pb}$ species desorbs upon adsorption of a new $\mathrm{Cd}$ species (Figure 7b). Repeating these two steps will lead to the replacement of $\mathrm{Pb}$ by $\mathrm{Cd}$ in an 
entire lattice plane, a process that will be facilitated by the hopping of $\mathrm{Cd}$ or $\mathrm{Pb}$ species over the QD surface (Figure 7e). Moreover, similar sequential hopping steps can bring vacancies deeper in the crystal, thus leading to the progressive transformation of $\mathrm{PbS}$ into $\mathrm{CdS}$.

The various process steps represented in Figure 7 imply that the QD surface has a double role. It should provide both the $\mathrm{Cd}$ species and the vacant surface sites for the cation exchange reaction to proceed. This is exactly what explains the anomalous behavior of the exchange experiments discussed here, where the cation exchange runs to completion for low $N_{\mathrm{Cd}}: N_{\mathrm{Pb}}$ ratios yet is strongly hampered at high $N_{\mathrm{Cd}}: N_{\mathrm{Pb}}$ ratios. In the latter case, the (dynamic) adsorption/desorption equilibrium of $\mathrm{Cd}$ species will be strongly shifted toward adsorption. This poisons the QD surface, ultimately leaving few surface sites transiently unoccupied, and thus impedes the cation exchange process. The favoring of desorption at low $N_{\mathrm{Cd}}: N_{\mathrm{Pb}}$ ratios on the other hand leads to the combination of surface sites occupied and unoccupied by $\mathrm{Cd}$ species, a situation that promotes the exchange reaction. We stress that this picture provides a comprehensive explanation of the experiments on both the $\mathrm{PbS}_{\mathrm{Cl}}$ and $\mathrm{PbS}_{\mathrm{TMS}} \mathrm{QDs}$. At high $N_{\mathrm{Cd}}: N_{\mathrm{Pb}}$ ratios, a largely similar shell thickness is obtained in spite of the differences in the initial exchange rate, suggesting that both have eventually a similar surface density of excess Cd. At low $N_{\mathrm{Cd}}: N_{\mathrm{Pb}}$ ratios and sufficiently high temperatures, the exchange can run to completion in both cases, which indicates that also for $\mathrm{PbS}_{\text {TMS }}$ empty surface sites become available. The fact that the exchange reaction runs to completion at a somewhat lower temperature with $\mathrm{PbS}_{\mathrm{Cl}} \mathrm{QDs}$ may reflect the differences in the initial density of excess cations on the surface or could result from subtle differences in surface chemistry, such as the presence of $\mathrm{Cl}^{-}$ions at the $\mathrm{PbS}_{\mathrm{Cl}}$ surface. In addition, the heterogeneity in exchange behavior of $\mathrm{PbS}_{\mathrm{Cl}}$ QDs, with part of them transforming completely in CdS, can be attributed within this picture to a considerable spread in initial surface termination by $\mathrm{Pb}$, which cannot be the case in $\mathrm{PbS}_{\mathrm{TMS}}$ QDs given their large $\mathrm{Pb}$ excess. This will result in large differences in exchange rates, with part of the QDs consuming all available $\mathrm{Cd}$ before others start to exchange.

\section{CONCLUSIONS}

In summary, we have investigated the role played by the QD surface in cation exchange reactions by means of the example of $\mathrm{Pb}$ for $\mathrm{Cd}$ exchange in $\mathrm{PbS}$ quantum dots. Using QDs that have either a full or a partial surface coverage by excess $\mathrm{Pb}$, we highlight the role played by vacant excess cation sites in the cation exchange process. They allow for the fast, initial adsorption of $\mathrm{Cd}$ species, and they enable the exchange of cations between the inner lattice sites of the quantum dot and its surface. In this way, the rate of a cation exchange reaction depends on the occupation of the surface by both excess $C d$ and vacant excess cation sites, similar to a bimolecular surface reaction. As a result, the cation exchange reaction will run to completion only at low $\mathrm{Cd}$ excess, when the PbS surface is not poisoned by excess surface $\mathrm{Cd}$. This understanding of the role of the surface reaction provides a key element needed for a unifying, mechanistic picture of cation exchange processes on nanocrystals that could account for the remarkable differences in exchange rate and be used for a more rational determination of optimal exchange conditions.

\section{METHODS}

$\mathrm{PbS}_{\mathrm{Cl}}$ Synthesis. $\mathrm{PbS}_{\mathrm{Cl}} \mathrm{QDs}$ of $5 \mathrm{~nm}$ were synthesized based on the procedure of Cademartiri et al..$^{30}$ and Moreels et al. ${ }^{28} \mathrm{~A}$ stock solution of $0.16 \mathrm{~g}(5 \mathrm{mmol})$ of $\mathrm{S}$ dissolved in $15 \mathrm{~mL}$ of oleylamine (OLA) is prepared by heating the mixture under nitrogen for $30 \mathrm{~min}$ at $120^{\circ} \mathrm{C}$. For the synthesis, $\left.0.834 \mathrm{~g} \mathrm{(3} \mathrm{mmol}\right)$ of $\mathrm{PbCl}_{2}$ and $7.5 \mathrm{~mL}$ of OLA were added to a three-neck flask. This is degassed for 30 min under nitrogen at $125^{\circ} \mathrm{C}$. Hereafter, we heat the $\mathrm{PbCl}_{2}$ solution to $130{ }^{\circ} \mathrm{C}$, and we inject $2.25 \mathrm{~mL}$ of the OIA-S stock solution. The temperature drops by approximately $10^{\circ} \mathrm{C}$, and the resulting growth temperature is maintained for $1 \mathrm{~min}$. The reaction is quenched by adding $10 \mathrm{~mL}$ of toluene and $15 \mathrm{~mL}$ of ethanol (EtOH). After centrifugation of the suspension and decantation of the supernatant, the QDs are resuspended in $6 \mathrm{~mL}$ of toluene.

In order to improve the stability of the QD dispersion, a ligand exchange was carried out from OLA to oleic acid (OA). An exchange to $O A$ is typically performed by adding $O A$ to a toluene suspension of PbS QDs in a ratio of 1.5:10 OA/toluene. After precipitation with EtOH and centrifugation, the QDs are resuspended in toluene and the exchange is repeated. Finally, the QDs are precipitated one more time with $\mathrm{EtOH}$ to remove any excess OA. Finally, QDs were dissolved in $10 \mathrm{~mL}$ of toluene and stored under an inert atmosphere.

$\mathrm{PbS}_{\mathrm{TMS}}$ Synthesis. PbS $\mathrm{PMS}_{\mathrm{TMS}} \mathrm{QDs}$ of $4.5 \mathrm{~nm}$ were synthesized based on the procedure developed by Abel et al., ${ }^{31}$ which was

carried out in a nitrogen-filled glovebox. Typically, $0.2232 \mathrm{~g}$ (1 $\mathrm{mmol}$ ) of $\mathrm{PbO}, 20 \mathrm{~mL}$ of diphenyl ether (DPE), and $1.3 \mathrm{~mL}$ $(4 \mathrm{mmol})$ of $\mathrm{OA}$ were mixed in a three-neck flask. This was heated at $120^{\circ} \mathrm{C}$ for $60 \mathrm{~min}$ to form a lead oleate complex (indicated by the transition of the initially yellow-colored solution to a colorless solution). Prior to the injection, the temperature was lowered to $118^{\circ} \mathrm{C}$. Following the rapid injection of a mixture of $8 \mu \mathrm{L}$ of bis(trimethylsilyl) sulfide (TMS) and $2 \mathrm{~mL}$ of trioctylphosphine, the temperature of the reaction mixture dropped to $110^{\circ} \mathrm{C}$. The particles were allowed to grow for $2 \mathrm{~min}$, after which the reaction was quenched by addition of $10 \mathrm{~mL}$ of butanol and $10 \mathrm{~mL}$ of methanol. The particles were collected by centrifugation, and the precipitate was washed with acetone and treated with an ultrasonicator for some time to remove any excess of OA. Finally, the obtained QDs were dissolved in $4 \mathrm{~mL}$ of toluene and stored under an inert atmosphere.

$\mathrm{PbS} / \mathrm{CdS}$ Core/Shell Synthesis. PbS/CdS core/shell QDs were synthesized starting from PbS core QDs by means of a cation exchange reaction initially proposed by Pietryga et al. ${ }^{12}$ For a standard reaction, cadmium oleate $(0.3 \mathrm{M}$ stock solution) is added in an atomic ratio of $\mathrm{Cd}: \mathrm{Pb}=20: 1$ to a $\mathrm{PbS}$ QD suspension in toluene heated to $100^{\circ} \mathrm{C}$. After reaching the desired reaction time, the reaction is quenched using a mixture of methanol and butanol (1:2), and the solution is purified twice. The final product is dissolved in toluene and stored under an inert 
atmosphere. The amount of cadmium oleate, temperature, and reaction time can be modified in order to obtain the desired shell thickness.

Core/Shell QDs' First Exciton Transition. The first exciton transition of $\mathrm{PbS} / \mathrm{CdS}$ core/shell QDs can become difficult to discern, especially for the thicker shells. We therefore fit the absorbance spectrum to a series of Gaussian spectra superimposed on a polynomial background. Subtracting the background from the absorption spectrum gives us a "net" absorption spectrum used to calculate the shell thickness.

RBS Measurements. RBS samples are prepared by drop-casting a small amount of PbS QDs on a MgO substrate, thereby forming a 100-200 nm close-packed thin film. The measurements are performed with a $2.5 \mathrm{MeV} \mathrm{He}^{+}$ion beam and two solid-state detectors placed at a backscattering angle of $165^{\circ}$ and $167.6^{\circ}$, respectively. From the backscattered yield, $R$ is determined:

$$
R=\frac{A_{\mathrm{Pb}}}{A_{\mathrm{S}}}\left(\frac{Z_{\mathrm{S}}}{Z_{\mathrm{Pb}}}\right)^{2}
$$

$A_{\mathrm{Pb}}$ denotes the area of the peak resulting from backscattering at $\mathrm{Pb}$ atoms, $A_{\mathrm{S}}$ equals the area of the $\mathrm{S}$ peak, and $Z_{\mathrm{Pb}}$ and $Z_{\mathrm{S}}$ are the atomic number of $\mathrm{Pb}$ and $\mathrm{S}$, respectively.

ICP-OES Measurements. Elemental analysis by means of ICPOES was used to determine the contents of $\mathrm{Pb}$ and $\mathrm{Cd}$ in a sample of core/shell QDs. From the results thus obtained, core diameter and shell thickness are calculated, assuming that the core/shell particle consists of a spherical core with a concentric, spherical shell. For elemental analysis, known amounts of $\mathrm{PbS} / \mathrm{CdS}$ QDs were dried in glass vials under a nitrogen gas flow, resuspended in $1.5 \mathrm{~mL}$ of aqua regia, and quantitatively transferred to Teflon vessels. Samples subsequently underwent a closed vessel microwave-assisted acid digestion procedure, using a Milestone Microwave Labstation MLS-1200 Mega (Milestone s.r.l., Italy). Finally, samples were diluted with ultrapure water to a volume of $15 \mathrm{~mL}$. Concentrations of $\mathrm{Pb}$ and $\mathrm{Cd}$ in external calibration standards ranged between 0.05 and $25 \mathrm{mg} / \mathrm{L}$ in 10\% (v/v) aqua regia. Selected emission lines were 220.353 and $168.215 \mathrm{~nm}$ for $\mathrm{Pb}$ and 214.438 and $226.502 \mathrm{~nm}$ for $\mathrm{Cd}$.

Conflict of Interest: The authors declare no competing financial interest.

Acknowledgment. This research has been funded by the FWO-Vlaanderen (G.0760.12), BelSPo (IAP 7.35, photonics@be), the FP7-STREP Navolchi, and BOF-UGent (GOA project). Dr. Karen Van Hoecke and Prof. Dr. Frank Vanhaecke (Atomic and Mass Spectrometry Unit, Ghent University) are acknowledged for the ICP-OES measurements. Vitaliy Bliznuk (Department of Materials Science and Engineering, Ghent University) is acknowledged for the STEM-HAADF images.

Supporting Information Available: The Supporting Information includes a TEM study on the morphology of the PbS and $\mathrm{PbS} / \mathrm{CdS} \mathrm{QDs}$, the formula for the $\mathrm{Pb}: \mathrm{S}$ excess at full surface coverage, the derivation of the formula for the maximum shell thickness for each $\mathrm{Cd}: \mathrm{Pb}$ ratio, the absorption coefficient of $\mathrm{PbS}$ / CdS QDs, a graph depicting the results and STEM-EDX spectroscopy measurements, and the quantification of the PbS/CdS and CdS QD fractions from the absorbance spectrum. This material is available free of charge via the Internet at http:// pubs.acs.org.

\section{REFERENCES AND NOTES}

1. Beberwyck, B. J.; Surendranath, Y.; Alivisatos, A. P. Cation Exchange: A Versatile Tool for Nanomaterials Synthesis. J. Phys. Chem. C 2013, 117, 19759-19770.

2. Rivest, J. B.; Jain, P. K. Cation Exchange on the Nanoscale: An Emerging Technique for New Material Synthesis, Device Fabrication and Chemical Sensing. Chem. Soc. Rev. 2013, 42, 89-96.

3. Chan, E. M.; Marcus, M. A.; Fakra, S.; ElNaggar, M.; Mathies, R. A.; Alivisatos, A. P. Millisecond Kinetics of Nanocrystal Cation Exchange Using Microfluidic X-Ray Absorption Spectroscopy. J. Phys. Chem. A 2007, 111, 12210-12215.
4. Luther, J. M.; Zheng, H.; Sadtler, B.; Alivisatos, A. P. Synthesis of PbS Nanorods and Other lonic Nanocrystals of Complex Morphology by Sequential Cation Exchange Reactions. J. Am. Chem. Soc. 2009, 131, 16851-16857.

5. Jain, P. K.; Amirav, L.; Aloni, S.; Alivisatos, A. P. Nanoheterostructure Cation Exchange: Anionic Framework Conservation. J. Am. Chem. Soc. 2010, 132, 9997-9999.

6. Li, H.; Zanella, M.; Genovese, A.; Povia, M.; Falqui, A.; Giannini, C.; Manna, L. Sequential Cation Exchange in Nanocrystals: Preservation of Crystal Phase and Formation of Metastable Phases. Nano Lett. 2011, 11, 4964-4970.

7. Casavola, M.; Huis, M. A. V.; Bals, S.; Lambert, K.; Hens, Z.; Vanmaekelbergh, D. Anisotropic Cation Exchange in PbSe/ CdSe Core/Shell Nanocrystals of Different Geometry. Chem. Mater. 2012, 24, 294-302.

8. Beberwyck, B. J.; Alivisatos, A. P. Ion Exchange Synthesis of III-V Nanocrystals. J. Am. Chem. Soc. 2012, 134, 1997719980.

9. Dong, C.; van Veggel, F. C. J. M. Cation Exchange in Lanthanide Fluoride Nanoparticles. ACS Nano 2009, 3, 123-130.

10. Son, D. H.; Hughes, S. M.; Yin, Y. D.; Alivisatos, A. P. Cation Exchange Reactions in lonic Nanocrystals. Science 2004, 306, 1009-1012.

11. Li, H.; Brescia, R.; Krahne, R.; Bertoni, G.; Alcocer, M. J. P.; Andrea, C. D.; Scotognella, F.; Tassone, F.; Zanella, M.; Giorgi, M. D.; et al. Blue-UV-Emitting ZnSe(Dot)/ ZnS(Rod) Core/Shell Nanocrystals Prepared from CdSe/CdS Nanocrystals by Sequential Cation Exchange. ACS Nano 2012, 6, 1637-1647.

12. Pietryga, J. M.; Werder, D. J.; Williams, D. J.; Casson, J. L.; Schaller, R. D.; Klimov, V. I.; Hollingsworth, J. A. Utilizing the Lability of Lead Selenide to Produce Heterostructured Nanocrystals with Bright, Stable Infrared Emission. J. Am. Chem. Soc. 2008, 130, 4879-4885.

13. Lambert, K.; Geyter, B. D.; Moreels, I.; Hens, Z.; De Geyter, B. $\mathrm{PbTe} / \mathrm{CdTe}$ Core/Shell Particles by Cation Exchange, a HRTEM Study. Chem. Mater. 2009, 21, 778-780.

14. Park, J.; Kim, S. W. CulnS 2 /ZnS Core/Shell Quantum Dots by Cation Exchange and their Blue-Shifted Photoluminescence. J. Mater. Chem. 2011, 21, 3745-3750.

15. Kim, S.; Kim, T.; Kang, M.; Kwak, S. K.; Yoo, T. W.; Park, L. S.; Yang, I.; Hwang, S.; Lee, J. E.; Kim, S. K.; et al. Highly Luminescent InP/GaP/ZnS Nanocrystals and their Application to White Light-Emitting Diodes. J. Am. Chem. Soc. 2012, 134, 3804-3809.

16. Robinson, R. D.; Sadtler, B.; Demchenko, D. O.; Erdonmez, C. K.; Wang, L. W.; Alivisatos, A. P. Spontaneous Superlattice Formation in Nanorods through Partial Cation Exchange. Science 2007, 317, 355-358.

17. Justo, Y.; Goris, B.; Kamal, J. S.; Geiregat, P.; Bals, S.; Hens, Z. Multiple Dot-in-Rod PbS/CdS Heterostructures with High Photoluminescence Quantum Yield in the Near-Infrared. J. Am. Chem. Soc. 2012, 134, 5484-5487.

18. Zhong, X.; Feng, Y.; Zhang, Y.; Gu, Z.; Zou, L. A Facile Route to Violet-to-Orange-Emitting $\mathrm{Cd}_{x} \mathrm{Zn}_{1-x}$ Se Alloy Nanocrystals via Cation Exchange Reaction. Nanotechnology 2007, 18, 385606.

19. Smith, A. M.; Nie, S. Bright and Compact Alloyed Quantum Dots with Broadly Tunable Near-Infrared Absorption and Fluorescence Spectra through Mercury Cation Exchange. J. Am. Chem. Soc. 2011, 133, 24-26.

20. Gupta, S.; Zhovtiuk, O.; Vaneski, A.; Lin, Y. C.; Chou, W. C.; Kershaw, S. V.; Rogach, A. L. $\mathrm{Cd}_{x} \mathrm{Hg}_{(1-x)}$ Te Alloy Colloidal Quantum Dots: Tuning Optical Properties from the Visible to Near-Infrared by lon Exchange. Part. Part. Syst. Charact. 2013, 30, 346-354.

21. Sahu, A.; Kang, M. S.; Kompch, A.; Notthoff, C.; Wills, A. W.; Deng, D.; Winterer, M.; Frisbie, C. D.; Norris, D. J. Electronic Impurity Doping in CdSe Nanocrystals. Nano Lett. 2012, 12, 2587-2594.

22. Eilers, J.; Groeneveld, E.; Donega, C. D. M.; Meijerink, A. Optical Properties of Mn-Doped ZnTe Magic Size Nanocrystals. J. Phys. Chem. Lett. 2012, 3, 1663-1667.

23. Kovalenko, M. V.; Talapin, D. V.; Loi, M. A.; Cordella, F.; Hesser, G.; Bodnarchuk, M. I.; Heiss, W. Quasi-Seeded 
Growth of Ligand-Tailored PbSe Nanocrystals through Cation-Exchange-Mediated Nucleation. Angew. Chem., Int. Ed. 2008, 47, 3029-3033.

24. Deka, S.; Miszta, K.; Dorfs, D.; Genovese, A.; Bertoni, G.; Manna, L. Octapod-Shaped Colloidal Nanocrystals of Cadmium Chalcogenides via "One-Pot" Cation Exchange and Seeded Growth. Nano Lett. 2010, 10, 3770-3776.

25. Ethayaraja, M.; Bandyopadhyaya, R. Model for Core/Shell Nanoparticle Formation by lon-Exchange Mechanism. Ind. Eng. Chem. Res. 2008, 47, 5982-5985.

26. White, S. L.; Smith, J. G.; Behl, M.; Jain, P. K. Co-operativity in a Nanocrystalline Solid-State Transition. Nat. Commun. 2013, 4, 2933-2941.

27. Routzahn, A. L.; Jain, P. K. Single-Nanocrystal Reaction Trajectories Reveal Sharp Cooperative Transitions. Nano Lett. 2014, 14, 987-992.

28. Moreels, I.; Justo, Y.; De Geyter, B.; Haustraete, K.; Martins, J. C.; Hens, Z. Size-Tunable, Bright, and Stable PbS Quantum Dots: A Surface Chemistry Study. ACS Nano 2011, 5 , 2004-2012.

29. Moreels, I.; Lambert, K.; De Muynck, D.; Vanhaecke, F.; Poelman, D.; Martins, J. C. J. C.; Allan, G.; Hens, Z. Composition and Size-Dependent Extinction Coefficient of Colloidal PbSe Quantum Dots. Chem. Mater. 2007, 19, 6101-6106.

30. Cademartiri, L.; Bertolotti, J.; Sapienza, R.; Wiersma, D. S.; von Freymann, G.; Ozin, G. A. Multigram Scale, Solventless, and Diffusion-Controlled Route to Highly Monodisperse PbS Nanocrystals. J. Phys. Chem. B 2006, 110, 671-673.

31. Abel, K. A.; Shan, J.; Boyer, J. C.; Harris, F.; Veggel, F. C. J.M. V. Highly Photoluminescent PbS Nanocrystals: The Beneficial Effect of Trioctylphosphine. Chem. Mater. 2008, 20, 37943796.

32. Justo, Y.; Geiregat, P.; Hoecke, K. V.; Vanhaecke, F.; De Mello Donega, C.; Hens, Z. Optical Properties of PbS/CdS Core/ Shell Quantum Dots. J. Phys. Chem. C 2013, 117, 2017120177.

33. Bartnik, A. C.; Wise, F. W.; Kigel, A.; Lifshitz, E. Electronic Structure of $\mathrm{PbSe} / \mathrm{PbS}$ Core-Shell Quantum Dots. Phys. Rev. B 2007, 75, 245424.

34. Grinbom, G. A.; Saraf, M.; Saguy, C.; Bartnik, A. C.; Wise, F.; Lifshitz, E. Density of States in a Single PbSe/PbS Core/ Shell Quantum Dot Measured by Scanning Tunneling Spectroscopy. Phys. Rev. B 2010, 81, 1-7.

35. Anderson, N. C.; Hendricks, M. P.; Choi, J. J.; Owen, J. S. Ligand Exchange and the Stoichiometry of Metal Chalcogenide Nanocrystals: Spectroscopic Observation of Facile Metal-Carboxylate Displacement and Binding. J. Am. Chem. Soc. 2013, 135, 18536-18548. 